\title{
Molar mass determination of lignins and characterization of their polymeric structures by multi-detector gel permeation chromatography
}

\author{
Evamaria C. Gaugler ${ }^{1 *}$ (D, Wolfgang Radke ${ }^{2}$, Andrew P. Vogt ${ }^{1}$ and Dawn A. Smith ${ }^{1}$
}

\begin{abstract}
Molar masses, Mark-Houwink-Sakurada (MHS) exponents, and refractive index increments (dn/dc) for three lignins were determined without derivatization by multi-detector gel permeation chromatography (GPC) in dimethylformamide (DMF) with $0.05 \mathrm{M}$ lithium bromide (LiBr). The lack of effectiveness of fluorescence filters on molar mass determination by GPC-multi-angle laser light scattering (MALS) was confirmed for softwood kraft lignin (Indulin AT) and revealed for mixed hardwood organosolv lignin (Alcell) as well as soda straw/grass lignin (Protobind 1000). GPC with viscometry detection confirmed that these lignins were present as compact molecules. The MHS exponent $a$ for Indulin AT and Alcell was in the order of 0.1. Additionally, the intrinsic viscosity of Protobind 1000 for a given molar mass was much lower than that of either Alcell or Indulin AT. This is the first report of $\mathrm{dn} / \mathrm{dc}$ values for these three lignins in DMF with $0.05 \mathrm{M} \mathrm{LiBr}$.
\end{abstract}

Keywords: Lignin analysis, Molecular weight, Gel permeation chromatography (GPC), Size exclusion chromatography (SEC)

\section{Introduction}

The molar mass of lignin is a key parameter which governs its reactivity and physical properties. The accurate determination of molar mass of lignins has posed a major challenge for a number of years for various reasons such as its highly complex and variable structure, and the lack of suitable standards (Baumberger et al., 2007). There are numerous publications describing gel permeation chromatography (GPC) methods for different types of lignins. Baumberger et al. gave a comprehensive summary on commonly used GPC conditions for the analysis of isolated industrial and model lignins (Baumberger et al., 2007). Tolbert et al. summarized current and future methodological developments used for determining the molecular weight of lignin from

\footnotetext{
* Correspondence: evamaria.gaugler@scionresearch.com

'Scion, Rotorua, New Zealand

Full list of author information is available at the end of the article
}

various sources at different points in the biorefining process (Tolbert et al., 2014). Liao et al. gave a conclusive review on current advancements on the isolation, characterization, and application of lignin (Liao et al., 2020). Andrianova et al. evaluated the applicability of size exclusion chromatographic systems and the effect of undesired secondary interactions to the lignin molar mass (Andrianova et al., 2018). Lange et al. set out to standardize experimental set-up and data treatment for GPC analysis of derivatized lignins in THF in combination with NMR, adding in a lignin-type (mainly in regard to linkage units p-hydroxyphenyl $(\mathrm{H})$, guaiacyl $(\mathrm{G})$, or syringyl (S)) dependent correction factor to render more precise molecular weight data (Lange et al., 2016). Most publications focus on organic GPC using tetrahydrofuran (THF) as an eluent and analyzing acetylated lignin (mainly kraft, organosolv, and milled wood lignin (MWL)) with ultraviolet (UV) detection at a wavelength 
of $280 \mathrm{~nm}$ (Baumberger et al., 2007; Glasser et al., 1993; Pellinen \& Salkinoja-Salonen, 1985; Gellerstedt, 1992; Gosselink et al., 2004). However, as reported by Cathala et al., THF is a poor solvent even for acetylated lignins, and new systems for molar mass determinations of lignins are desirable (Cathala et al., 2003). Gidh et al. characterized lignin produced after purification from Kraft black liquor in water, dimethylsulfoxide (DMSO), and $0.1 \mathrm{M} \mathrm{NaOH}$ (Gidh et al., 2006). As an alternative to $\mathrm{THF}$, the use of $\mathrm{N}, \mathrm{N}$-dimethylformamide (DMF) with and without the addition of lithium bromide $(\mathrm{LiBr})$ has been reported in literature for molar mass determination of lignin (Tolbert et al., 2014; Gosselink et al., 2004; Cathala et al., 2003; Connors et al., 1980; Dong \& Fricke, 1995). Many lignins are readily soluble in DMF, and derivatization such as acetylation, acetobromination, and benzoylation of the lignin is not required. This is beneficial as derivatization is a time-consuming procedure that changes the functionality of the lignin. Additionally, many high molar mass lignin fractions are not readily soluble in the derivatization reagent, and functionalized lignins cannot be derivatized without losing the functional groups. As DMF is well-known for promoting association effects resulting in bimodal elution profiles (Cathala et al., 2003; Connors et al., 1980), the addition of lithium chloride or bromide to DMF was recommended to minimize aggregation and to obtain monomodal elution curves (Cathala et al., 2003).

Conventional GPC employing a single concentration detector, such as a refractive index (RI) or ultraviolet (UV) detector, is most commonly used for the determination of the relative molar mass of lignins (Baumberger et al., 2007). This is done by comparing their elution volume against that of standards with known molar mass. If the standards used in the calibration and the sample are of the same chemical and topological structure, then, accurate molar masses can be obtained. Unfortunately, lignin standards are not commercially available, and therefore, other calibrants are usually applied. Andrianova et al. addressed the issue of calibration standards by investigating the suitability of four sets of commercially available polymeric standards as well as low molar mass lignin model compounds on several GPC column sets with different functional groups, confirming minimal stationary phase interactions except for size exclusion effects. They confirmed that both poly(styrene) (PS) and poly(methyl methacrylate) (PMMA) standards can be used for accurate GPC column calibration (Andrianova et al., 2018). However, if the standards and the sample are dissimilar molecular structures, the results are merely comparative. In multi-detector GPC, a viscometer, or a light scattering detector, or both are employed together with a concentration detector to address the limitations of conventional GPC. When applying GPC with molar mass-sensitive detectors (such as a viscometer or a light-scattering detector), absolute molar mass and molar mass distribution can be calculated that is not dependent on the chemistry of any standards used in calibration. In addition, use of molar mass-sensitive detectors allows the determination of other polymer properties that cannot be obtained by conventional GPC.

A viscometer measures the specific viscosity of materials as they elute from the column. Combining this with concentration information from a RI or UV detector allows determination of the intrinsic viscosity, which in turn allows for molar mass determination at each elution volume based on the universal calibration concept (Grubisic et al., 1967). Since both, molar mass and intrinsic viscosity, are known across the chromatogram, significant insights into the behavior of polymer molecules in solution can be obtained.

Using viscometry, the value of $\alpha$ in the Kuhn-MarkHouwink-Sakurada plot (often referred to as just the Mark-Houwink-Sakurada (MHS) plot) gives information about the structural behavior of the polymer molecules in solution. Values of $\alpha$ between 0 and 0.5 indicate a compact dense structure (following the "hard sphere" model). Values of $\alpha$ of $0.7-0.8$ are expected for linear random coils in a good solvent, and even larger values of $\alpha$ are found for larger, more solvated structures. An upper limit of 2.0 is indicative of the "rigid rod" model, where most of the segments of the molecule are far from the center of gravity, similar to that expected for a spinning rod structure in solution. Thus, the MHS relationship is an excellent way of probing the structure of polymer molecules in solution (Technologies, 2017). Dong et al. reported MHS parameters for kraft lignin in DMF at two temperatures $\left(\alpha=0.11\right.$ at $45{ }^{\circ} \mathrm{C}$ and $\alpha=$ 0.13 at $78^{\circ} \mathrm{C}$ ) (Dong \& Fricke, 1995). Additionally, MHS parameters for different types of lignins in solvents such as THF, dimethylformamide, acetone, and sodium hydroxide solutions have been reported (Glasser et al., 1993; Cathala et al., 2003; Ringena et al., 2006). All these investigations showed low $\alpha$ values well below 0.5 , indicating dense structures.

Using a light-scattering detector to determine absolute molar mass of lignins is challenging because fluorescence interferes strongly and leads to overestimation of the molar mass (Cathala et al., 2003; Froment \& Pla, 1989; Merkle et al., 1992). However, reports in literature are incongruous regarding the efficiency and role of fluorescence filters and if light scattering provides true molar masses for lignin. For example, Siochi et al. reported that in order to use low-angle laser light scattering (LALLS) detection, one has to consider not only the influence of the sample absorbance and fluorescence on molecular weight determination but also the effect of 
beam polarization (Siochi et al., 1990). Fredheim et al. stressed the importance of including fluorescence filters in the multi-angle laser light scattering (MALS) detector in the study of lignosulfonates, even when using a laser at $632.8 \mathrm{~nm}$ (Fredheim et al., 2002). In the present study, the effect of fluorescence filtering on the molar masses of softwood kraft lignin (Indulin AT), a hardwood organosolv lignin (Alcell), and a soda straw/grass lignin (Protobind 1000) in DMF with $0.05 \mathrm{M} \mathrm{LiBr}$ was investigated by GPC-MALS.

Knowledge of the sample refractive index increment $(\mathrm{dn} / \mathrm{dc})$ in the eluent is a requirement for the molar mass determination by GPC using MALS and viscometry detection. Contreras et al. determined $\mathrm{dn} / \mathrm{dc}$ values of $0.140 \pm 0.011$ and $0.165 \pm 0.008 \mathrm{~mL} \mathrm{~g}^{-1}$ for Norway spruce enzymatic mild acidolysis lignin (EMAL) and $\mathrm{Eu}$ calyptus globulus (E. globulus) EMAL, for freshly dissolved acetobrominated samples in THF (Contreras et al., 2008). Siochi et al. measured $\mathrm{dn} / \mathrm{dc}$ values of hydroxypropyl (HP) derivatives of a red oak organosolv lignin, an aspen organosolv lignin, and a Westvaco hardwood kraft lignin freshly dissolved in THF (Siochi et al., 1990). The determined $\mathrm{dn} / \mathrm{dc}$ values were $0.145 \pm$ $0.016,0.152 \pm 0.017$, and $0.163 \pm 0.023 \mathrm{~mL} \mathrm{~g}^{-1}$, respectively. To the best of our knowledge, $\mathrm{dn} / \mathrm{dc}$ values for Indulin AT, Alcell, and Protobind 1000 in DMF with $0.05 \mathrm{M} \mathrm{LiBr}$ have not been reported yet.

Molar mass distributions of lignin in solutions are influenced by not only the type of solvents used but also by the time in solution (incubation time) otherwise called the effect of aging (Glasser et al., 1993; Guerra et al., 2007). Guerra et al. observed a dependency of molecular weight distribution upon incubation time for acetobrominated EMAL from Southern pine and Eucalyptus globulus solubilized in THF (Guerra et al., 2007). Samples were analyzed after 5, 10, and 20 days. Glasser et al. found when examining the effect of aging on Indulin AT Kraft lignin acetate and Eucalin Eucalyptus hardwood lignin in THF that there were variations between the lignin types (Glasser et al., 1993). The molar mass data was constant for the Eucalin sample as was the number average molar mass $(\mathrm{Mn})$ for the Indulin AT lignin acetate. However, the weight average molar mass (Mw) of Indulin AT exhibited a 16\% reduction after 3 days and further reductions with longer times. The steady reductions in $\mathrm{Mw}$ were explained by lignin acetate associations being disrupted with time (Glasser et al., 1993). However, no information has been found in literature on the aging effect of lignins in $0.05 \mathrm{M}$ $\mathrm{LiBr}$ in DMF. Hence, the influence of incubation time on the molar mass distribution of Indulin AT solubilized in $0.05 \mathrm{M} \mathrm{LiBr}$ in DMF solution was investigated over the duration of $48 \mathrm{~h}$.

Using concentration detectors (UV and RI) in combination with a calibration curve, it is known that the sample concentration can influence molar mass evaluation by GPC (Hofe, 2007; Striegel et al., 2009). Large injected masses could lead to column overloading and reduced separation efficiency resulting in increasing errors in the average molar masses. The influence of sample concentration on the molar mass determination of Indulin AT, Alcell, and Protobind was evaluated to choose the best suited concentration for the concentration detector (RI) in combination with viscometer and MALS detector.

To the best of our knowledge, multi-detector GPC, using both MALS and viscometry detection, has not previously been used to determine molar masses of lignins and their polymeric structure in DMF with the addition of $0.05 \mathrm{M} \mathrm{LiBr}$. This study aims to fill this gap by employing multi-detector GPC to determine molar masses as well as MHS exponent $\alpha$ and $\mathrm{dn} / \mathrm{dc}$ values for a softwood (G-type) kraft lignin (Indulin AT), a hardwood (S- and G-type) organosolv lignin (Alcell), and a soda straw/grass (S, G and H-type) lignin (Protobind 1000) (Constant et al., 2016). In addition, the effectiveness of fluorescence filters for MALS detector measurements was investigated.

\section{Experimental section \\ Chemicals and solvents}

The lignins investigated were Indulin AT (MeadWestvaco, USA), Alcell (Repap Technologies Inc, USA), and Protobind 1000 (Asian Lignin Manufacturing Private Limited, India) (see Table 1). Alcell and Protobind 1000 were received from the International Lignin Institute (ILI) (Switzerland). DMF, for high-performance liquid chromatography (HPLC), $\geq 99.9 \%$; and LiBr anhydrous, beads, $\sim 10$ Mesh, $\geq 99.9 \%$ trace metal basis were purchased from Sigma-Aldrich (New Zealand). Toluene LiChrosolv, for HPLC, $\geq 99.9 \%$ purchased from Merck (New Zealand), was used as an internal flow marker.

A narrow-distributed PS standard certified as European Reference Material (ERM FA001) by BAM (Federal Institute for Materials Research and Testing, Berlin, Germany) was used to perform a detector set-up. The standard had a certified molar mass $\mathrm{Mw}$ of $87,600 \mathrm{~g} \mathrm{~mol}^{-1}$.

A PMMA ReadyCal Kit consisting of 12 standards (Polymer Standards Service (PSS), Germany) in the molar mass range between 800 and 988,000 $\mathrm{g} \mathrm{mol}^{-1}$ was used for column calibration.

Table 1 Sample descriptions

\begin{tabular}{ll}
\hline Lignin & Description \\
\hline Indulin AT & Softwood kraft lignin \\
Alcell & Mixed hardwood organosolv lignin \\
Protobind 1000 & Soda lignin from straw/grass (Constant et al., 2016) \\
\hline
\end{tabular}




\section{GPC instrumentation}

Experiments were performed on 2 different GPC systems. Detector set-ups were performed for both systems using the narrow-distributed PS standard (ERM FA001) to determine the MALS detector instrument constants as well as the normalization coefficient for every angle and the interdetector delays and response factors for the concentration detectors (RI and UV). A dn/dc of 0.159 $\mathrm{mL} \mathrm{g}^{-1}$ was applied for this standard in $0.05 \mathrm{M} \mathrm{LiBr}$ in DMF at $55{ }^{\circ} \mathrm{C}$. More details regarding detector set-up is given in the supplementary information.

\section{GPC system 1}

The multi-detector molar mass determinations were performed on a GPC system consisting of the following Smartline (Knauer, Germany) and SECcurity (PSS, Germany) components: a pump (Smartline 1050), a 2channel inline degasser (SECcurity), an autosampler (Smartline 3950), a column thermostat (Smartline), a RI detector using light-emitting diode (LED) light with a wavelength of $950 \mathrm{~nm}$ (Smartline 2300), an UV detector (Smartline 2550), a viscosity detector (SECcurity ETA2010), and an online MALS detector with a red laser using a wavelength of $637 \mathrm{~nm}$ (SECcurity SLD 7100). The MALS detector worked simultaneously at 7 angles and was equipped with a fluorescence filter. The fluorescence filter had a center wavelength of $637.5 \mathrm{~nm}$ with a bandwidth of $13 \mathrm{~nm}$.

\section{GPC system 2}

The effectiveness of fluorescence filters during GPCMALS measurements were investigated for Indulin AT and Alcell on a GPC system at the laboratory of PSS in Mainz (Germany). A SECcurity GPC system (PSS, Germany) equipped with pump, autosampler, RI detector using LED light with a wavelength of $950 \mathrm{~nm}$, and an online MALS detector with a red laser using a wavelength of $637 \mathrm{~nm}$ (SECcurity SLD 7100) was used. Two different SDL7100 MALS instruments were applied, where one instrument was equipped with a fluorescence filter, while the other was not. Samples were analyzed in duplicate.

\section{Column sets}

For both systems, the separations were performed on a GRAM column set consisting of a $10 \mu \mathrm{m}$ GRAM guard column $(50 \times 8 \mathrm{~mm})$ and two $10 \mu \mathrm{m}$ GRAM linear columns $(300 \times 8 \mathrm{~mm})$ in Lux quality for light-scattering experiments (PSS, Germany). The column separation range was between 500 and 1,000,000 $\mathrm{g} \mathrm{mol}^{-1}$. Eluent was $0.05 \mathrm{M} \mathrm{LiBr}$ in DMF at $55{ }^{\circ} \mathrm{C}$ at a flow rate of 1.0 $\mathrm{mL} \mathrm{min}^{-1}$. An injection volume of $100 \mu \mathrm{L}$ was used.

\section{Procedures}

\section{Sample preparation}

GPC samples were prepared (in triplicate) in $0.05 \mathrm{M}$ $\mathrm{LiBr}$ in DMF solution. Sample solutions for GPC had lignin concentrations ranging between 0.3 and $6.0 \mathrm{mg}$ $\mathrm{mL}^{-1}$. Standards were prepared to $2.0 \mathrm{mg} \mathrm{mL} \mathrm{m}^{-1}$. Lignins revealed a very low viscosity, due to their compact structure (see below). Therefore, multi-detector (RI, UV, MALS, and viscometry) GPC experiments were performed using a concentration of $6.0 \mathrm{mg} \mathrm{mL}^{-1}$, to obtain a suitable signal/noise ratio for the viscosity detector. For conventional GPC experiments, a concentration of $1.5 \mathrm{mg} \mathrm{mL}{ }^{-1}$ was chosen. The samples were dissolved under agitation at room temperature and analyzed by GPC within $2 \mathrm{~h}$ unless otherwise stated. Prior to use, solutions were filtered through $0.45-\mu \mathrm{m}$ hydrophilic polytetrafluoroethylene (PTFE) syringe filters (Hydraflon, MicroAnalytix, New Zealand). Toluene was added to the prepared samples and the standards as an internal standard to define the end of integration and to detect possible system fluctuations (column aging, flow rate fluctuations, leakages, etc.).

\section{Column calibration}

Column calibration was performed using PMMA standards (PSS, Germany). PMMA is the recommended polymer standard in DMF as explained in the supplementary information (Andrianova et al., 2018; Kuo et al., 1991; Coffey \& Harmer, 2015; Dubin et al., 1977). A dn/dc of $0.061 \mathrm{~mL} \mathrm{~g}^{-1}$ was applied for the PMMA standards in $0.05 \mathrm{M} \mathrm{LiBr}$ in DMF at $55{ }^{\circ} \mathrm{C}$. A conventional calibration curve was established by plotting the elution volume of the PMMA standards against the log molar mass. A fourth-order polynomial fit function provided an excellent fit $\left(R^{2}=0.9999\right)$. It described the calibration points well, especially at the low molar mass end, where the major part of the samples eluted. Care was taken to avoid overfitting. The calibration data and curve are given in the supplementary information (Table s1 and Figure s1).

A universal calibration curve was established by measuring intrinsic viscosities of the PMMA standards via the viscometer. The log of the product of intrinsic viscosity $[\eta]$ and molar mass $M$ was plotted versus the retention volume. The $[\eta] M$ product is related to the hydrodynamic volume (Grubisic et al., 1967). A fourth order polynomial fit function provided an excellent fit $\left(R^{2}=0.9999\right)$. The MHS parameters ( $K$ and $\alpha$ ) for the PMMA standards, calculated with the WinGPC UniChrom software (PSS, Germany), were $K=0.019$ and $\alpha=0.71$.

\section{Data evaluation}

For the purpose of sample evaluation, the baseline and the integration limits were applied in a way to include all sample eluting from the column even the high molar 
mass agglomerates, if present. The baseline was applied from 12.5 to $31.5 \mathrm{~mL}$. The low molar mass integration limit was set to $25.8 \mathrm{~mL}$ which marks the beginning of the elution of the low molar mass marker toluene. The high molar mass integration limit was adjusted individually for the different lignins between 12.9 and $20.0 \mathrm{~mL}$.

The WinGPC UniChrom software (PSS, Germany) was used for recording and evaluating light scattering, viscosity, and RI and UV profiles. Molar masses, weight average molar mass $(\mathrm{Mw})$ and number average molar mass $(\mathrm{Mn})$, were calculated from the RI detector response. For both MALS and viscometry detection, the slice concentration was determined based on the measured RI intensity, the RI instrument constant determined during detector setup, and the experimentally determined dn/dc values (Note, 2010; Wyatt, 1993). A basic equation for concentration or $\mathrm{dn} / \mathrm{dc}$ calculation is given in the supplementary information.

\section{Determination of the refractive index increment ( $d n / d c)$}

The $\mathrm{dn} / \mathrm{dc}$ values for the three lignins were determined by measuring a concentration series consisting of 6 samples with concentrations ranging between 0.3 and 6.0 $\mathrm{mg} \mathrm{mL} \mathrm{m}^{-1}$ for each lignin using RI detection (LED light with a wavelength of $950 \mathrm{~nm}$ ).
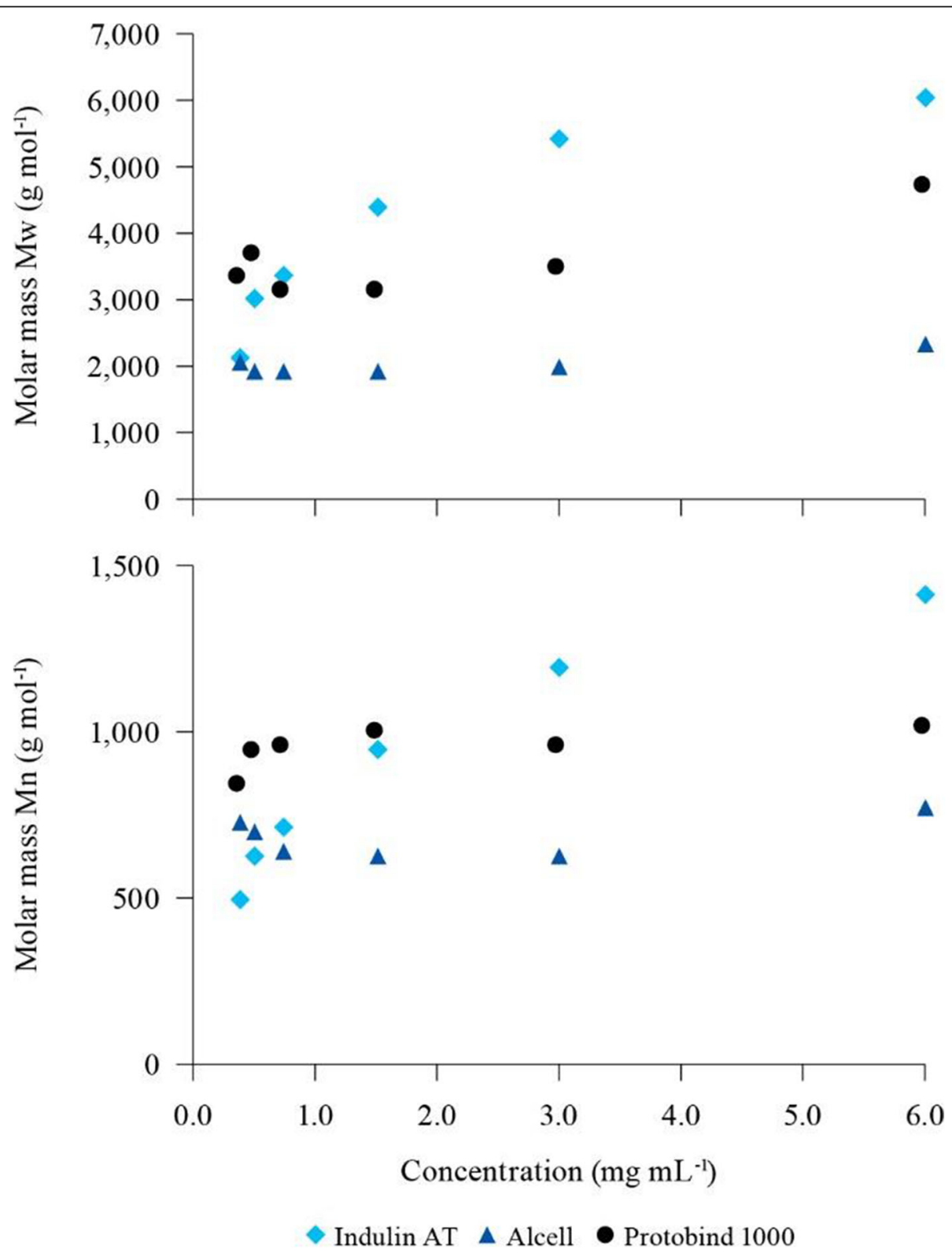

Fig. 1 Comparison of molar mass dependencies on the elution volume for Alcell with and without fluorescence filter 


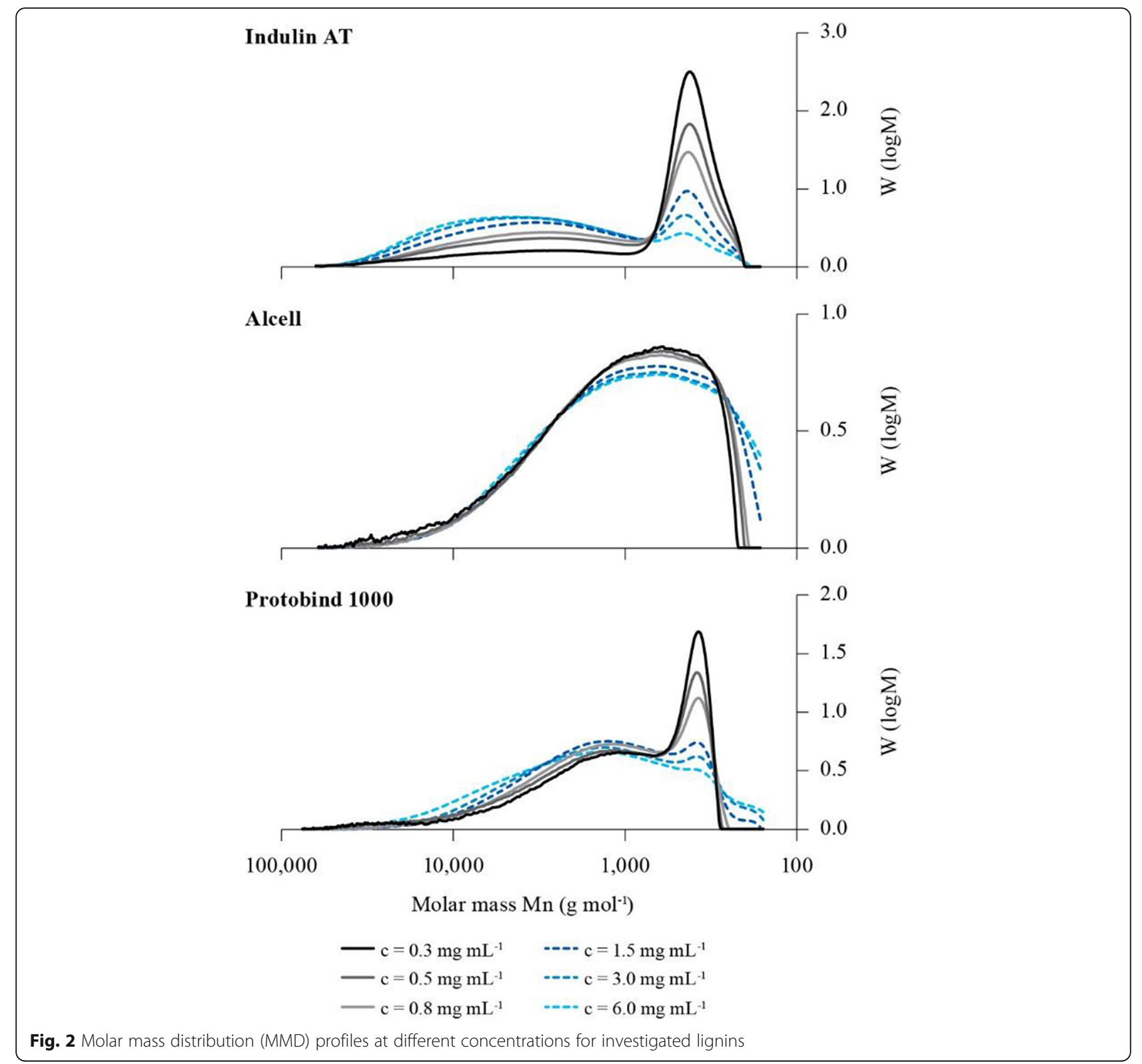

The area for the six concentrations was plotted versus the injected mass, and the $\mathrm{dn} / \mathrm{dc}$ was determined from the slope which reflects the detector constant multiplied by the dn/dc. The WinGPC UniChrom software (PSS, Germany) calculated $\mathrm{dn} / \mathrm{dc}$ values for a concentration series using the "detector linearity test" function.

The $\mathrm{dn} / \mathrm{dc}$ values for PS and PMMA standards in 0.05 $\mathrm{M} \mathrm{LiBr}$ in DMF at $55{ }^{\circ} \mathrm{C}$ were determined experimentally in the laboratory of Prof. Brent S. Sumerlin (Department of Chemistry, Southern Methodist University, Dallas, USA). More detail regarding experimental set-up is given in the supplementary information.

\section{Determination of MHS exponent a}

The MHS exponent $\alpha$ of the samples was determined by manually plotting $\log [\eta]$ versus $\log M$ for the individual GPC slices. The slope of the plot is $\alpha$. MHS parameters

Table 2 Molar mass for Indulin AT at various incubation times using Rl detection and a conventional calibration $(c=1.5 \mathrm{mg}$ $\mathrm{mL}^{-1}$ )

\begin{tabular}{|c|c|c|c|c|}
\hline Sample & Incubation time (h) & $\mathrm{Mn}\left(\mathrm{g} \mathrm{mol}^{-1}\right)$ & $\mathrm{Mw}\left(\mathrm{g} \mathrm{mol}^{-1}\right)$ & D \\
\hline Indulin AT & 0 & 1630 & 4580 & 2.8 \\
\hline Indulin AT & 24 & 1680 & 4730 & 2.8 \\
\hline Indulin AT & 48 & 1640 & 4730 & 2.9 \\
\hline
\end{tabular}




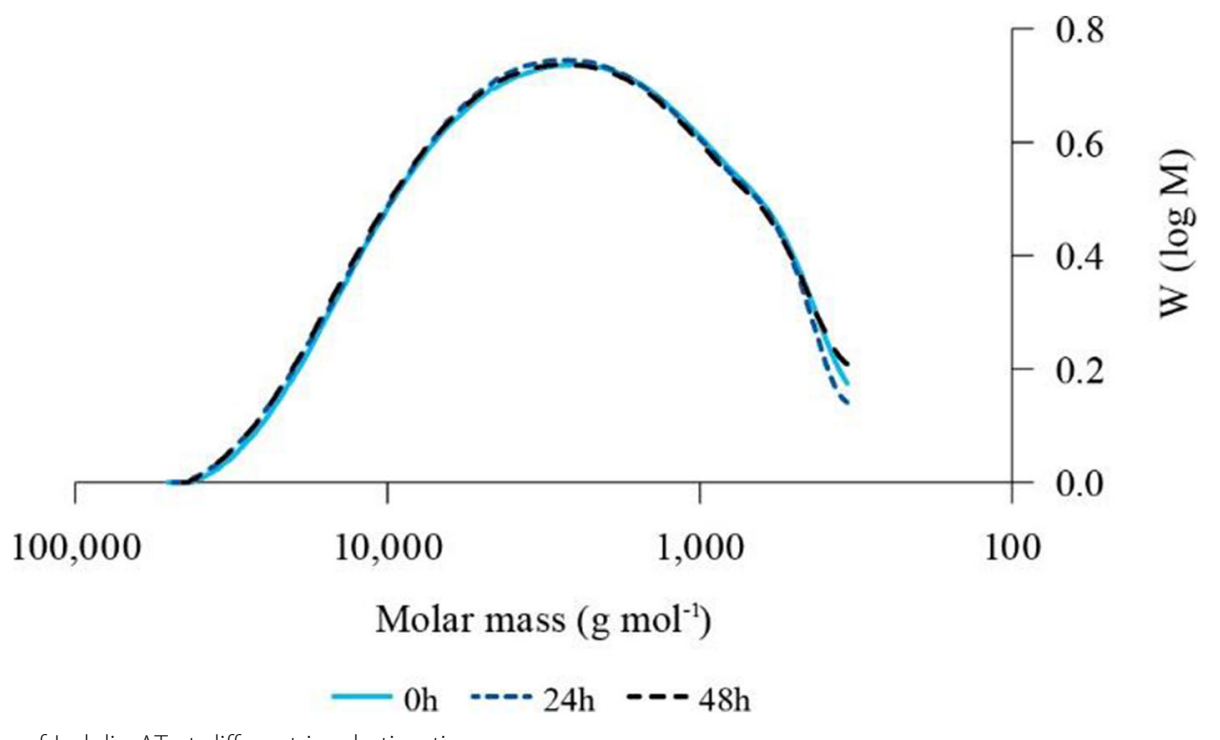

Fig. 3 MMD profiles of Indulin AT at different incubation times

( $K$ and $\alpha$ ) of PMMA in the eluent, required to establish a universal calibration curve, were calculated from the linear fit of the MHS plot in the calibration window in the WinGPC UniChrom software (PSS, Germany).

\section{Results and discussion}

\section{System evaluation}

\section{Influence of sample concentration}

The influence of sample concentration on the molar mass determination of various types of lignin was investigated using RI detection. For this purpose, lignins were analyzed using six different concentrations ranging between 0.3 and $6.0 \mathrm{mg} \mathrm{mL}^{-1}$. The molar masses $(\mathrm{Mn}$ and $\mathrm{Mw}$ ), determined by RI detection using a conventional PMMA calibration curve, were plotted versus the injected sample concentrations to determine their concentration dependency (Figs. 1 and 2). The concentration dependencies of molar masses differed for the various types of lignins. The number average molar masses (Mn) of Indulin AT increased with increasing sample concentration from $490 \mathrm{~g} \mathrm{~mol}^{-1}$ at $0.3 \mathrm{~mL}^{-1}$ to $1410 \mathrm{~g} \mathrm{~mol}^{-1}$ at $6.0 \mathrm{~mL}^{-1}$. The weight average molar masses $(\mathrm{Mw})$ of Indulin AT increased with increasing sample concentration from $2100 \mathrm{~g} \mathrm{~mol}^{-1}$ at $0.3 \mathrm{~mL}^{-1}$ to $6020 \mathrm{~g} \mathrm{~mol}^{-1}$ at $6.0 \mathrm{~mL}^{-1}$ (Figs. 1 and 2). Values for the number average molar mass $(\mathrm{Mn})$ and weight average molar mass $(\mathrm{Mw})$ for Indulin AT increased about three times. For Alcell, no significant effect of concentration on the number and weight average molar masses was observed. The number molar masses $(\mathrm{Mn})$ of Alcell remained in the range between 700 and $760 \mathrm{~g} \mathrm{~mol}^{-1}$ and the weight average molar masses $(\mathrm{Mw})$ between 1880 and $2280 \mathrm{~g} \mathrm{~mol}^{-1}$ independent of the concentration. Sample concentrations below $6.0 \mathrm{mg} \mathrm{mL}^{-1}$ did not affect the derived number and weight average molar masses (Mn and Mw) of Protobind 1000. In contrast, the weight average molar mass $(\mathrm{Mw})$ of Protobind at a concentration of $6.0 \mathrm{mg} \mathrm{mL}^{-1}$ was affected by the sample concentration. It was about 1.5 times higher at 6.0 than at 0.8 $\mathrm{mg} \mathrm{mL}^{-1}$ (increasing from 3040 to $4650 \mathrm{~g} \mathrm{~mol}^{-1}$ ).

Typically, elution volumes become higher leading to lower molar masses with increasing concentration. However, in this case, a peak at lower elution volume increases with concentration due to aggregation, especially in the case of Indulin AT. The elugrams for the concentration series for Indulin AT indicate a growing bimodality with concentration. This can be expected as the concentration of Indulin AT is increased as the $\mathrm{LiBr}$ concentration remains constant. Connors et al. showed the extreme case of GPC of Indulin AT with no $\mathrm{LiBr}$ in DMF (bimodal) versus in $0.1 \mathrm{M} \mathrm{LiBr}$ in DMF (monomodal) (Connors et al., 1980). For Alcell, the concentration series only shows increased detector response with concentration. For Protobind 1000, the elugram becomes much broader at $6.0 \mathrm{mg} \mathrm{mL}^{-1}$, which is likely an indication of aggregation. This behavior emphasizes the importance of choosing the sample concentration carefully and stresses the difference between the investigated lignins. The general recommendation is to keep the sample concentration as low and as consistent as possible to

Table 3 Molar mass using MALS detection derived with and without fluorescence filter $\left(n=2 ; c=1.5 \mathrm{mg} \mathrm{mL}^{-1}\right)$

\begin{tabular}{lll}
\hline Sample & Mw-no filter $\left(\mathbf{g ~ m o l}^{\mathbf{- 1}}\right)$ & Mw-filter $(\mathbf{g ~ m o l}$ \\
\hline PS & 214,000 & 213,500 \\
Indulin AT & 319,000 & 44,600 \\
Alcell & 827,000 & 65,600 \\
\hline
\end{tabular}


avoid this effect (Dong \& Fricke, 1995). It is generally recognized that the sample concentration has to be chosen to suit the lignin type, the eluent, and the employed GPC detectors. Glasser et al. used concentrations ranging between 3.0 and $6.0 \mathrm{mg} \mathrm{mL}^{-1}$ for various types of lignin acetates in THF employing RI detection (Glasser et al., 1993). Zinovyev et al. dissolved their lignin samples in DMSO $\operatorname{LiBr}(0.5 \% \mathrm{w} / \mathrm{v})$ at $10.0 \mathrm{mg} \mathrm{mL}^{-1}$ for analysis using GPC-MALS (Zinovyev et al., 2018). For the analysis of lignins by multi-detector GPC including viscometry detection, a concentration of $6.0 \mathrm{mg}$ $\mathrm{mL}^{-1}$ was the lowest suitable concentration to obtain good $\mathrm{S} / \mathrm{N}$ ratios. However, such a high concentration will result in overestimation of the molar mass of Indulin AT and possibly Protobind 1000. This issue could potentially be overcome with the development of more sensitive viscosity detectors.

\section{Influence of incubation time}

The influence of incubation time on the molar mass distribution of Indulin AT solubilized in $0.05 \mathrm{M} \mathrm{LiBr}$ in DMF solution was investigated over the duration of 48 h. The molar mass of the sample relative to a conventional PMMA calibration curve was determined immediately after dissolution (T0), after $24 \mathrm{~h}$ (T1), and after 48 h (T2) at room temperature. The average molar masses, dispersities, and molar mass distributions are summarized in Table 2.

The average molar masses and dispersity of Indulin AT samples did not change over a period of $48 \mathrm{~h}$ suggesting that the molar mass is independent on timing of exposure in $0.05 \mathrm{M} \mathrm{LiBr}$ in DMF (Table 2, Fig. 3). Such independence is beneficial for establishing a reliable and reproducible GPC method. This is in contrast to the results previously reported in literature on the aging of acetylated or acetobrominated lignins in THF (Glasser et al., 1993; Contreras et al., 2008; Guerra et al., 2007). The aging of derivatized lignins in THF may be either due to lack of stability of derivatized products or the formation of reactive peroxides in unstabilized THF. However, further investigations using other lignins and increased incubation times are needed to confirm this finding.

\section{Influence of autofluorescence}

The effect of fluorescence filtering on the molar masses of lignin was investigated by GPC-MALS. For this purpose, two light-scattering instruments, one of which was equipped with a fluorescence filter, were applied. To account for the effect of different sensitivities at the photodiodes and optical alignments of both light-scattering instruments, a PS sample was analyzed to compare RI and $90^{\circ}$ light-scattering intensities. As expected, the RI peaks were nearly independent of the installed MALS unit. In addition, the intensities of the $90^{\circ}$ lightscattering signal for PS were very similar. Consequently, as expected, the installation of a fluorescence filter did not affect the molar mass determination of PS. The weight average molar mass (Mw) of PS was independent of the filter installation, remaining at 214,000 $\mathrm{g} \mathrm{mol}^{-1}$ (Table 3, Fig. 4). In contrast, application of a fluorescence filter strongly diminishes the $90^{\circ}$ light-scattering

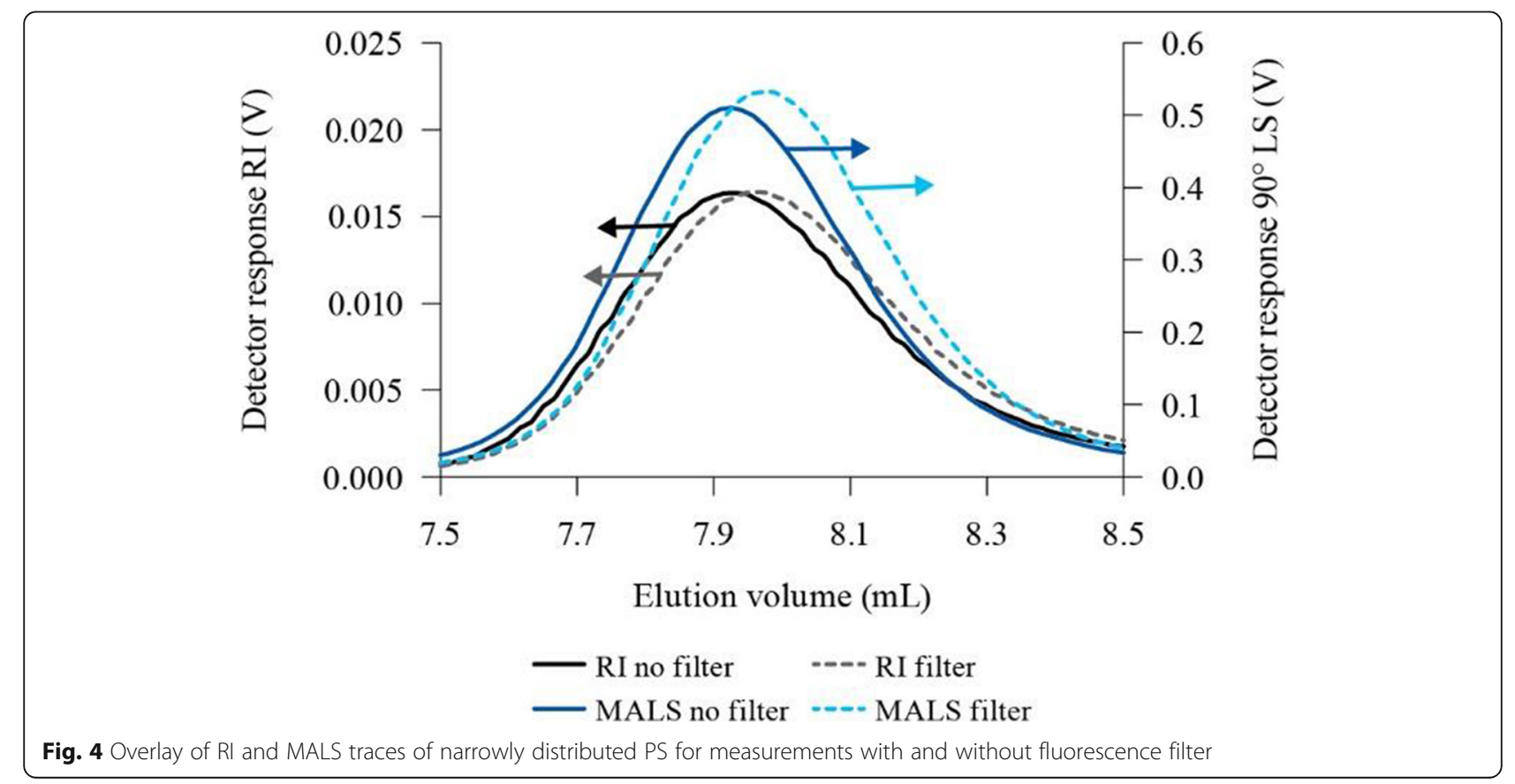


signals of lignin samples (Fig. 5), which results in much lower values for the apparent $\mathrm{Mw}$ of Indulin AT and Alcell at each elution volume. Consequently, the apparent weight average molar masses are reduced as well, as shown in Table 3. A reduction in apparent weight average molar mass $(\mathrm{Mw})$ of about 86\% (from 319,000 to 44,600 $\mathrm{g} \mathrm{mol}^{-1}$ ) was achieved for Indulin AT when using a fluorescence filter. The reduction was even higher for Alcell $\left(92 \%\right.$, from 827,000 to $\left.65,600 \mathrm{~g} \mathrm{~mol}^{-1}\right)$. However, a comparison of the molar masses derived by GPCMALS even with fluorescence filtering (Table 3) shows that these are still much higher than the ones obtained by either RI or viscometry detection (Tables 2 and 5). This discrepancy is discussed below together with the molar mass versus elution volume dependencies.

When plotting elution volumes versus molar masses determined by MALS detection, it was observed that the molar mass versus elution volume dependencies deviated from normal behavior for both lignins, Indulin AT and Alcell (Figs. 6 and 7). Though application of the fluorescence filter significantly reduced the apparent molar mass, it appeared as if the molar mass was constant or even increased with increasing elution volume. This gives the impression that the GPC separation mechanism was not operating correctly; however, the unexpected shape of the molar mass vs. elution volume curve is probably a consequence of the incomplete elimination of fluorescence. In contrast, for PS, the molar mass dependence was as expected for a GPC-like separation, and the molar mass decreased with increasing elution volume (Fig. 8), irrespective of the installation of a fluorescence filter. It was assumed that the phenomena observed with Indulin AT and Alcell was a consequence of residual fluorescence of the lignins.
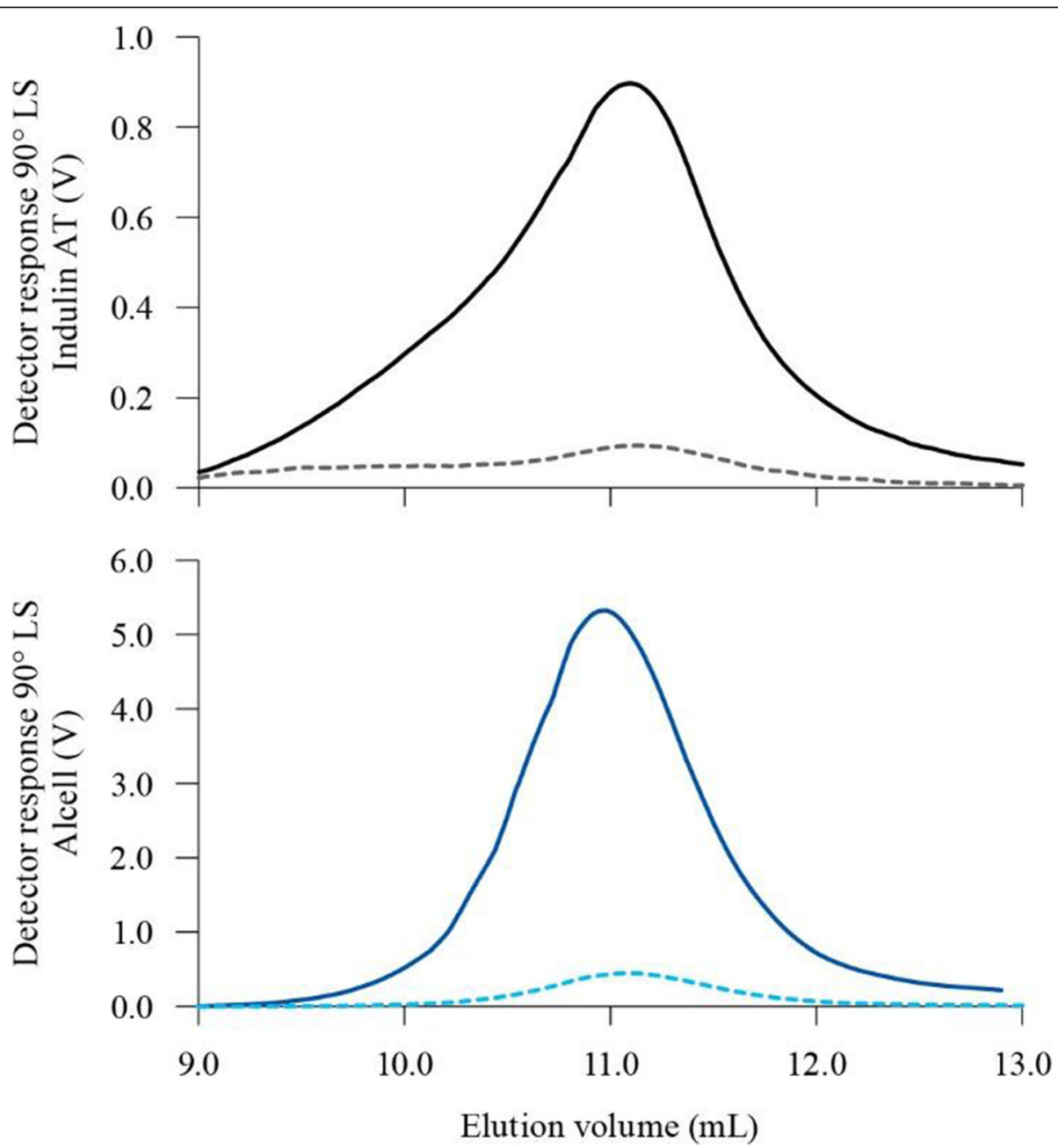

$\begin{array}{ll}\text { - Indulin AT no filter } & \text {---- Indulin AT filter } \\ \text { - Alcell no filter } & ---- \text { Alcell filter }\end{array}$

Fig. 5 Overlay of MALS traces of lignins Indulin AT and Alcell for measurements with and without fluorescence filter 


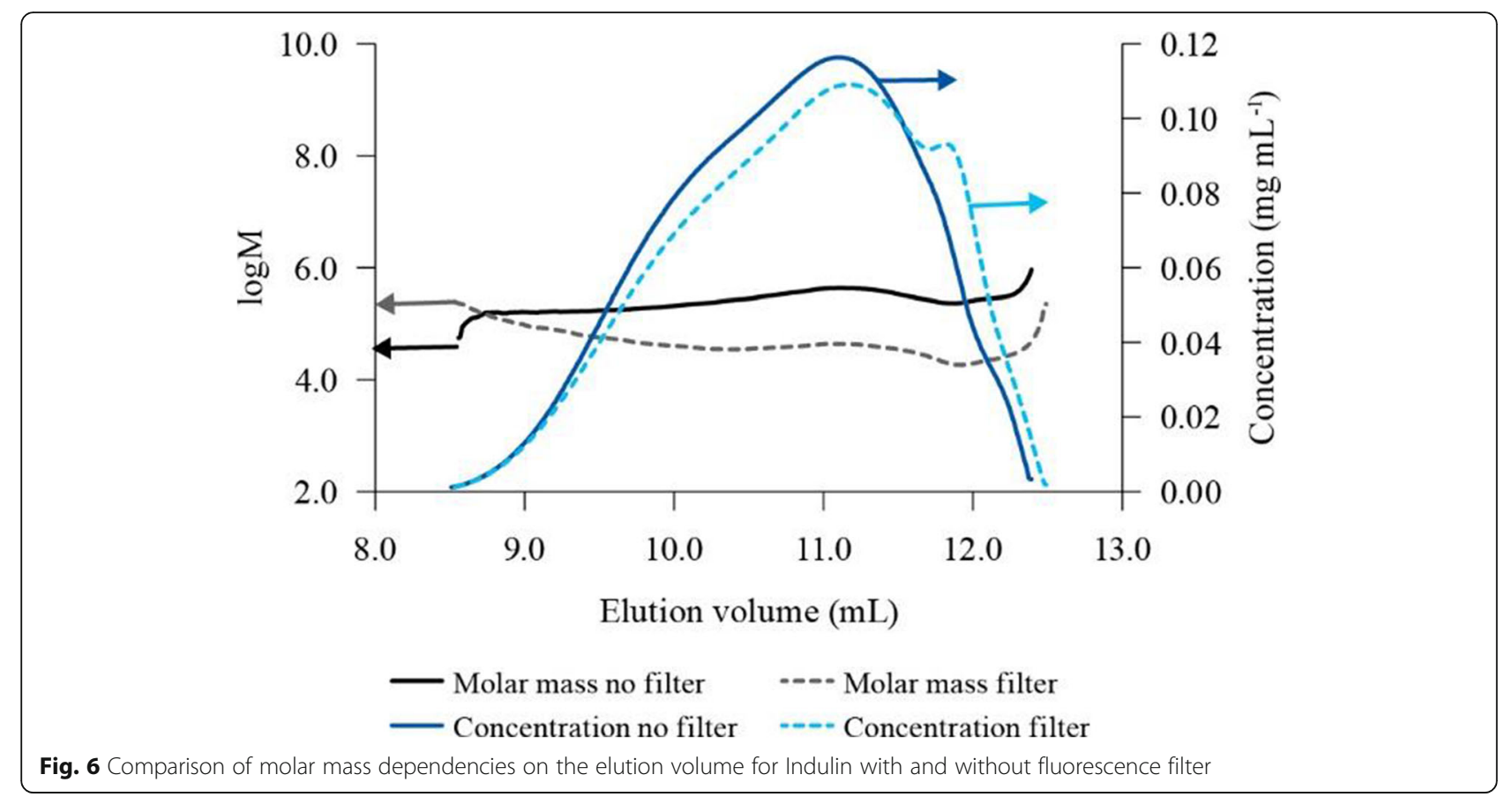

An explanation for the different effectivities of fluorescence filters on the molar mass of lignins is that only a certain wavelength-dependent fraction of the fluorescence intensity will be eliminated, while the residual percentage of the fluorescent light passes through the filter. Consequently, for highly fluorescent samples, there is a relatively high amount of non-absorbed fluorescent light passing through the filter which interferes with the true scattering intensity. If the intensity of this residual fluorescent light is comparable or significantly higher than the true scattering intensity, an incorrect apparent molar mass will be derived because the detected light intensity is dominated by fluorescence. Additionally, the fluorescence intensity depends on the wavelength of the MALS detector. With increasing wavelength, the probability of fluorescence decreases. To clearly identify the minimum wavelength

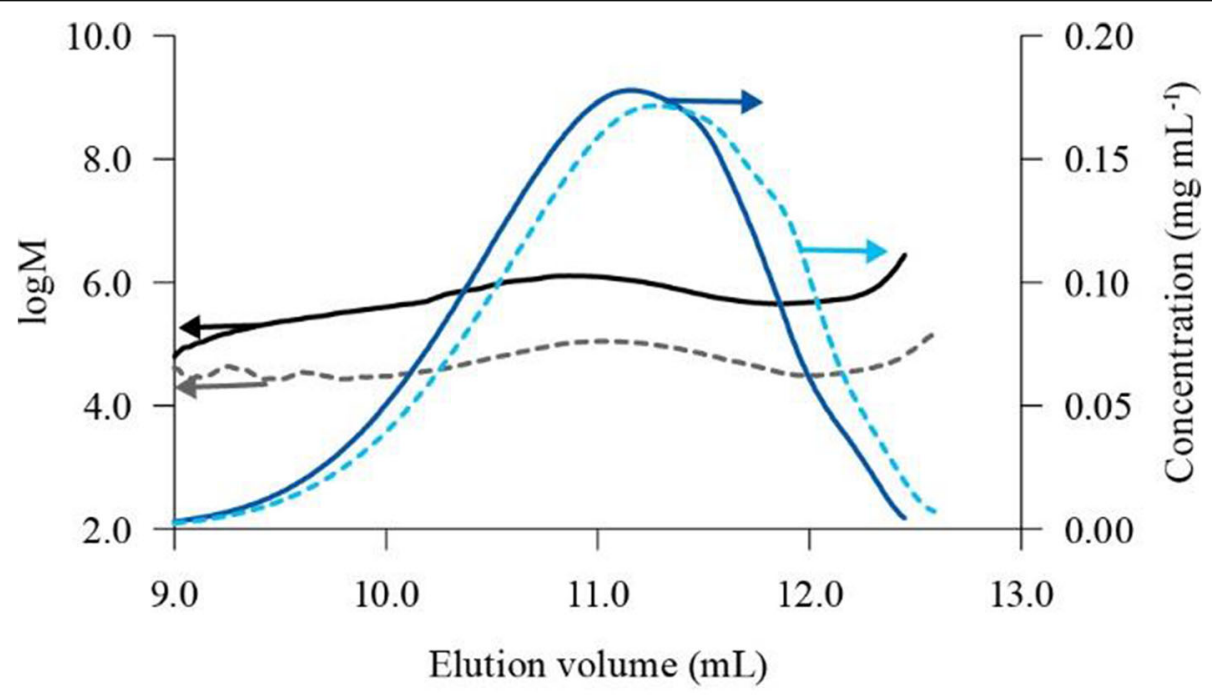

$\begin{array}{ll}\text { - Molar mass no filter } & ---- \text { Molar mass filter } \\ \text { - Concentration no filter } & ---- \text { Concentration filter }\end{array}$

Fig. 7 Comparison of molar mass dependencies on the elution volume for Alcell with and without fluorescence filter 


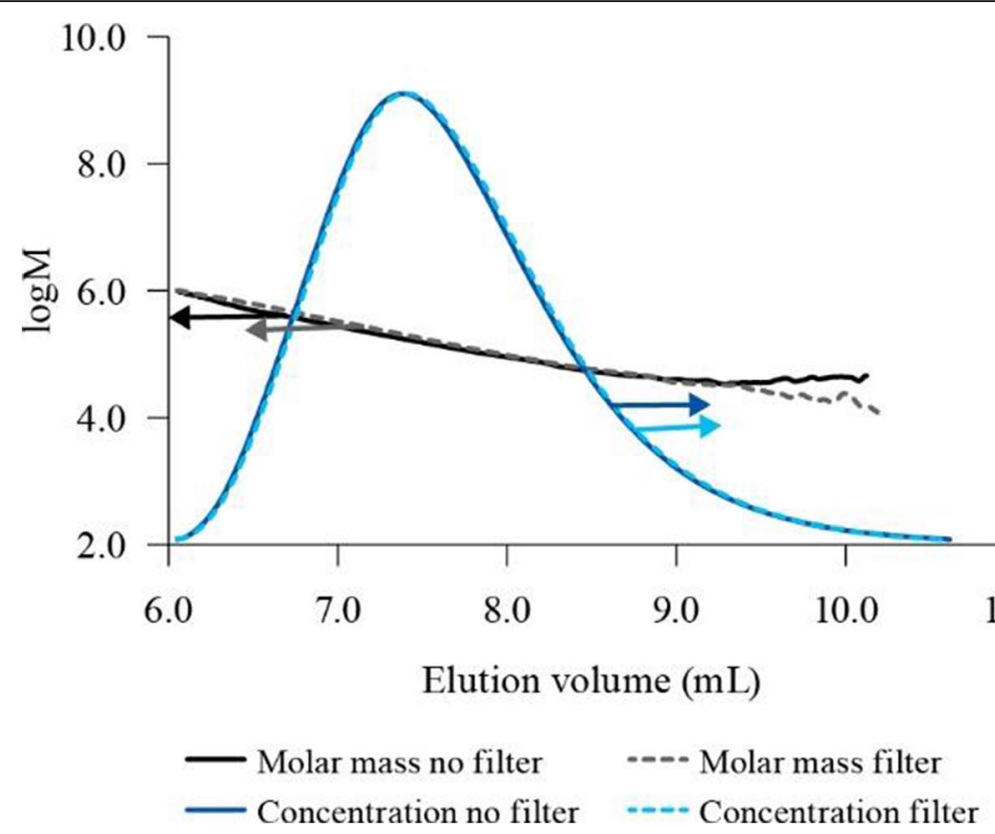

Fig. 8 Comparison of molar mass dependencies on the elution volume for measurements of broadly distributed PS with and without fluorescence filter

providing absence of fluorescence requires measuring molar masses at different wavelengths. Fluorescence is absent if identical molar masses are obtained for measurements at a minimum of two significantly different wavelengths. However, a minimum wavelength must be identified for each lignin type individually because the relative contribution of fluorescence to detected light scattering intensity depends on the sample itself.

In conclusion, whether a fluorescence filter is suitable to determine reliable molar masses depends on the sample characteristics. Simply introducing a fluorescence filter will not guarantee obtaining correct molar masses. This is in line with and explains recently published results by Zinovyev et al. (Zinovyev et al., 2018). They stated that MALS instruments with commonly available red lasers $(633,658$, or $690 \mathrm{~nm})$ equipped with a fluorescence filter were only sufficient for select lignosulfonates and mildly extracted lignins, not for Kraft lignins (Zinovyev et al., 2018). As part of this study, we were able to report that MALS instruments with a red laser equipped with a fluorescence filter are also not sufficient for mixed hardwood organosolv lignin (Alcell) as well as soda straw/grass lignin (Protobind 1000).

\section{Characterization of various lignins via multi-detector GPC Determination of $d n / d c$ values}

Knowledge of the sample $\mathrm{dn} / \mathrm{dc}$ in the eluent is a requirement for the molar mass determination by GPC using MALS and viscometry detection. The determined $\mathrm{dn} / \mathrm{dc}$ values for the three lignins in $0.05 \mathrm{M}$ $\mathrm{LiBr}$ in $\mathrm{DMF}$ at $55{ }^{\circ} \mathrm{C}$ ranged between 0.106 and
$0.134 \mathrm{~mL} \mathrm{~g}^{-1}$ (Table 4). The lowest $\mathrm{dn} / \mathrm{dc}$ value was measured for Protobind 1000 and the highest for Indulin AT, respectively. Differences in $\mathrm{dn} / \mathrm{dc}$ for lignins of different origin are consistent with the results of other investigators. Independent of the type of lignin, dn/dc values in $0.05 \mathrm{M} \mathrm{LiBr}$ in DMF at $55^{\circ} \mathrm{C}$ reported here were lower than the values reported for lignins in THF (Siochi et al., 1990; Contreras et al., 2008). Siochi et al. measured $\mathrm{dn} / \mathrm{dc}$ values of $0.145 \pm$ $0.016 \mathrm{~mL} \mathrm{~g}^{-1}$ for hydroxypropylated red oak organosolv lignin, $0.152 \pm 0.017 \mathrm{~mL} \mathrm{~g}^{-1}$ for hydroxypropylated aspen organosolv lignin, and $0.163 \pm 0.023 \mathrm{~mL}$ $\mathrm{g}^{-1}$ for hydroxypropylated Westvaco hardwood kraft lignin in THF (Siochi et al., 1990). Contreras et al. determined $\mathrm{dn} / \mathrm{dc}$ values of $0.140 \pm 0.0011 \mathrm{~mL} \mathrm{~g}$ for EMAL Norway spruce lignin and $0.165 \pm 0.008$ $\mathrm{mL} \mathrm{g}^{-1}$ for EMAL Eucalyptus globulus lignin in THF (Contreras et al., 2008). The lower $\mathrm{dn} / \mathrm{dc}$ values in DMF are expected because as a first approximation, the $\mathrm{dn} / \mathrm{dc}$ is proportional to the refractive index difference between the polymer and its solvent (Huglin, 1972). As the refractive index of THF is lower than that of DMF, dn/dc values of lignins in DMF are expected to be lower than those in THF. In addition,

Table $4 \mathrm{dn} / \mathrm{dc}$ values for investigated lignins

\begin{tabular}{lll}
\hline Sample & $\mathbf{d n} / \mathbf{d c}\left(\mathbf{m L ~ g}^{-\mathbf{1}}\right)$ & $\boldsymbol{R}^{\mathbf{2}}$ \\
\hline Indulin AT & 0.134 & 0.9995 \\
Alcell & 0.133 & 0.9995 \\
Protobind 1000 & 0.106 & 0.9980 \\
\hline
\end{tabular}


Table 5 Multi-detector molar mass ( \pm st dev) for investigated lignins $\left(n=3, c=6.0 \mathrm{mg} \mathrm{mL}^{-1}\right.$ )

\begin{tabular}{|c|c|c|c|c|}
\hline Detector/calibration & Sample & $\mathrm{Mn}\left(\mathrm{g} \mathrm{mol}^{-1}\right)$ & $M w\left(\mathrm{~g} \mathrm{~mol}^{-1}\right)$ & $D$ \\
\hline Rl/conventional & Indulin AT & $1750 \pm 100$ & $5600 \pm 270$ & $3.2 \pm 0.0$ \\
\hline Viscometry/universal & Indulin AT & $790 \pm 40$ & $6970 \pm 460$ & $8.8 \pm 0.3$ \\
\hline RI/conventional & Alcell & $980 \pm 0$ & $3130 \pm 30$ & $3.2 \pm 0.0$ \\
\hline Viscometry/universal & Alcell & $410 \pm 10$ & $3650 \pm 180$ & $9.0 \pm 0.2$ \\
\hline Rl/conventional & Protobind 1000 & $940 \pm 10$ & $3500 \pm 40$ & $3.7 \pm 0.1$ \\
\hline Viscometry/universal & Protobind 1000 & $430 \pm 30$ & $5690 \pm 200$ & $13.2 \pm 0.6$ \\
\hline
\end{tabular}

both Siochi et al. and Contreras et al. used different types of lignins. To the best of our knowledge, this is the first report of $\mathrm{dn} / \mathrm{dc}$ values for Indulin $\mathrm{AT}$, Alcell, and Protobind 1000 in DMF with $0.05 \mathrm{M} \mathrm{LiBr}$.

Experimentally determined $\mathrm{dn} / \mathrm{dc}$ values in $0.05 \mathrm{M}$ $\mathrm{LiBr}$ in DMF at $55{ }^{\circ} \mathrm{C}$ were used further in this study for the determination of molar mass of lignins by MALS and viscometry detection.

\section{Molar mass determination}

Molar masses of various lignins determined by multidetector GPC using viscometry and MALS detection were compared with conventional GPC using RI as a concentration detector.

The analysis of lignins by MALS detection resulted in much higher Mw values (between 4 and 29 times) for all three lignins even though the MALS detector was fitted with a fluorescence filter. The overestimation of molecular mass values was explained by residual fluorescence, as discussed in the "Influence of autofluorescence" section. For this reason, molar mass values determined by
MALS detection in the present study are only given in the supplementary information (Table s2) for comparative purposes.

The molar mass determination of the three lignins by viscometry, based on a universal calibration curve, resulted in higher weight average molar mass $(\mathrm{Mw})$ values (by 1.2 to 1.6 times) compared to RI detection using a conventional calibration curve (Table 5). Protobind 1000 showed the biggest increase in weight average molar mass $(\mathrm{Mw})$ from $3500 \pm 40$ to $5690 \pm 200 \mathrm{~g} \mathrm{~mol}^{-1}$. In contrast, number average molar mass $(\mathrm{Mn})$ values derived by viscosity detection were lower (by 2.2 to 2.4 times) for all lignins compared to RI detection (Table 5). Alcell showed the biggest decrease in number average molar mass $(\mathrm{Mn})$ from $980 \pm 0$ to $410 \pm 10 \mathrm{~g} \mathrm{~mol}^{-1}$. As a result, the dispersities resulting from conventional GPC are significantly lower than those obtained by GPC using viscometry detection. Assuming lignin structures did not change greatly across the molar mass distribution, the observed higher dispersities are consistent with a steeper lignin calibration curve and consequently with

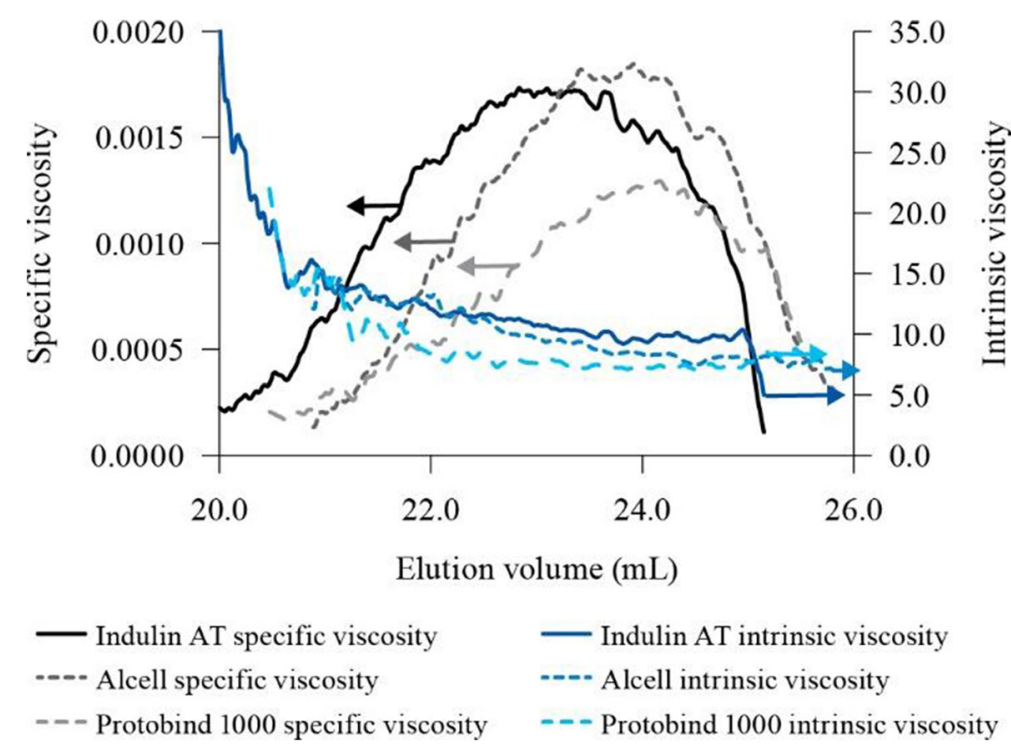

Fig. 9 Comparison of measured viscosities of Indulin AT, Alcell, and Protobind 1000 as a function of the elution volume 
a lower MHS exponent $\alpha$ compared to PMMA. A lower MHS exponent $\alpha$ indicates a more sphere-like structure of lignin compared to the coil-like structure of PMMA.

The benefit of using viscometry instead of MALS detection is that the viscosity and RI signals were not influenced by fluorescence. When plotting the intrinsic viscosity as a function of elution volume (Fig. 9), the expected decreasing intrinsic viscosities with elution volume were observed. However, for each lignin, differences in intrinsic viscosities at the same elution volume can be observed. This indicates that these lignins have different segment densities and therefore that different lignin structures elute at the same elution volume.

\section{Evaluation of polymeric structure}

By using viscometry detection, insights into the polymeric structure of materials in solution were obtained via the Mark-Houwink-Sakurada plot. For Indulin and Alcell, nearly linear dependencies of intrinsic viscosity on molar mass were observed (Fig. 10), allowing reliable determination of the MHS exponent $\alpha$ (Table 6). The MHS exponent $\alpha$ for Indulin AT and Alcell were of the order of 0.1. Thus, the scaling behavior of these samples in $0.05 \mathrm{M} \mathrm{LiBr}$ in DMF at $55{ }^{\circ} \mathrm{C}$ is close to a solid sphere. This is expected due to the more highly branched and condensed structures found in these lignins (Constant et al., 2016; Wang et al., 2020). Reported literature values for the MHS exponent $\alpha$ for various lignins in THF with and without the addition of LiBr (Liao et al., 2020; Pellinen \& Salkinoja-Salonen, 1985) were slightly higher. Cathala et al. (Cathala et al., 2003) reported $\alpha$ values for spruce MWL and guaiacyl lignin polymer models (G-DHPs) in THF with and without the addition of LiBr as ranging between 0.18 and 0.28. $\alpha$ values for 11 acetylated commercial lignins in THF varying between 0.17 and 0.35 were reported by Glasser et al. (Glasser et al., 1993). They also reported $\alpha$ values of 0.29 and 0.21 for Indulin AT and Alcell in THF, respectively.

High scatter in the data prevented assessment on the dependency of intrinsic viscosity on molar mass for Protobind 1000 (Fig. 10). A reason cannot be given at this point. Nonetheless, it was apparent that the slope was higher, indicating a less dense structure than Indulin AT and Alcell (Fig. 10). This more linear structure found in Protobind 1000 is likely due to the presence of $\mathrm{H}$-type lignin and less branching linkages per 100 aromatic units than seen in Indulin AT and Alcell (Constant et al., 2016). A double logarithmic plot of intrinsic viscosity versus molar mass of the three lignins in triplicate measurements is given in the supplementary information (Figure s2).

\section{Recommendations and conclusions}

This work provides valuable insight and information on the molar mass determination of three lignins and their polymeric structure by using multi-detector GPC, including molar mass sensitive detection, in $0.05 \mathrm{M} \mathrm{LiBr}$

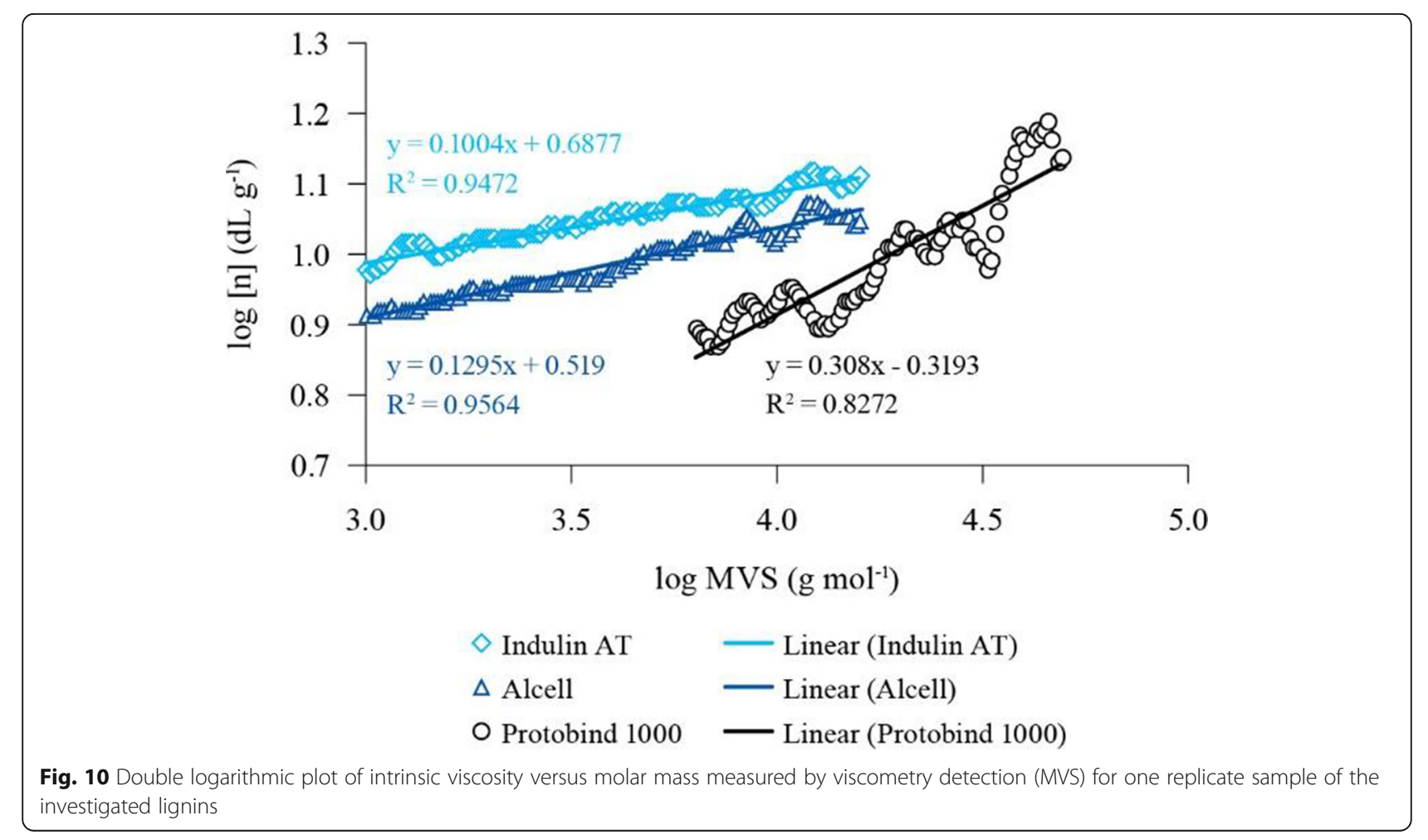


Table 6 MHS exponent a ( \pm st dev) for investigated lignins ( $n=$ 3)

\begin{tabular}{ll}
\hline Sample & MHS a \\
\hline Indulin AT & $0.09 \pm 0.03$ \\
Alcell & $0.13 \pm 0.04$ \\
\hline
\end{tabular}

in DMF. The lignins included a softwood (G-type) kraft lignin (Indulin AT), a hardwood (S- and G-type) organosolv lignin (Alcell), and a soda straw/grass (S, G, and Htype) lignin (Protobind 1000).

DMF with $\mathrm{LiBr}$ was found to be a suitable solvent for lignins for several reasons. Firstly, the three different lignins were all readily soluble in $0.05 \mathrm{M} \mathrm{LiBr}$ in DMF, and secondly, the molar mass profile of Indulin AT lignin was stable and did not change on aging over a $48 \mathrm{~h}$ period. The independence of molar mass on time in solution is beneficial for establishing a reliable and reproducible GPC method. This work confirmed that the MALS detection resulted in overestimation of the molar masses even though a fluorescence filter has been used. This was explained by residual fluorescence. MALS detectors using commonly available red laser $(633,658$, or $690 \mathrm{~nm}$ ) equipped with a fluorescence filter are not sufficient for absolute molar mass determination of Indulin AT, Alcell, and Protobind 1000 in $0.05 \mathrm{M} \mathrm{LiBr}$ in DMF solution. It was concluded that the suitability of a fluorescence filter for the reliable determination of molar masses depends on the sample characteristics. Simply introducing a fluorescence filter will not guarantee obtaining correct molar masses. Even though it is known that the application of longer wavelengths in MALS detectors can result in additional reduction of fluorescence, the success of such approaches depends on the fluorescence strength of the samples themselves. Therefore, further work is needed to determine at which wavelength fluorescence is sufficiently reduced to allow for reliable molar mass determination of lignins irrespective of lignin origin and type.

As to our knowledge, this is the first report of $\mathrm{dn} /$ dc and MHS exponent $\alpha$ values as well as molar masses using viscometry detection for three lignins in $0.05 \mathrm{M} \mathrm{LiBr}$ in DMF. Using GPC with viscometry detection confirmed that Indulin AT and Alcell are present as very compact molecules in $0.05 \mathrm{M} \mathrm{LiBr}$ in DMF which result from the inherent structural differences found in these different lignins. In this work a concentration of $6.0 \mathrm{mg} \mathrm{mL}^{-1}$ was used for determining the molar masses using viscometry detection to obtain good $\mathrm{S} / \mathrm{N}$ ratios. However, the study confirmed that such a high concentration will result in overestimation of the molar mass of Indulin AT and possibly Protobind 1000. A future research challenge is to improve the $\mathrm{S} / \mathrm{N}$ ratio when using viscometry detection without overestimating the molar mass. This issue could potentially be overcome with the development of more sensitive viscometers.

\section{Abbreviations}

DMF: Dimethylformamide; DMSO: Dimethylsulfoxide; Dn/dc: Refractive index increments; EMAL: Enzymatic mild acidolysis lignin; ERM: European Reference Material; GPC: Gel permeation chromatography; HP: Hydroxypropyl; HPLC: High-performance liquid chromatography; HVMS: High-value manufacturing and services; ILI: International Lignin Institute; LALLS: Lowangle laser light scattering; LED: Light-emitting diode; LiBr: Lithium bromide; MALS: Multi-angle laser light scattering; Mn: Number average molar mass; MVS: Molar mass measured by viscometry detection; Mw: Weight average molar mass; MWL: Milled wood lignin; PMMA: Poly(methyl methacrylate); PS: Poly(styrene); PSS: Polymer Standards Service; RI: Refractive index; PTFE: Polytetrafluoroethylene; SEC: Size exclusion chromatography; THF: Tetrahydrofuran; UV: Ultraviolet

\section{Supplementary Information}

The online version contains supplementary material available at https://doi. org/10.1186/s40543-021-00283-5.

Additional file 1: Supporting information.

\section{Acknowledgements}

The expert advice regarding general GPC of Dr Michael Krämer (PSS, Germany) is gratefully acknowledged. The authors would like to thank Dr Alfred Abächerli (ILI, Switzerland) for providing us with lignin reference samples as well as sharing his expertise in lignin analysis via multi-detector GPC. The authors would like to thank the New Zealand Ministry of Business, Innovation, and Employment funding under HVMS Enabling Technologies investment contract C04X1205, Extrusion Plus for funding the work.

\section{Authors' contributions}

ECG, WR, APV, and DAS conceived the study. ECG, WR, and APV contributed to the experimental design. ECG, WR, and APC performed the multi-detector GPC analysis of lignin, PS, and PMMA. ECG, WR, APV, and DAS wrote the manuscript. The authors read and approved the final manuscript.

\section{Funding}

This work was supported by the New Zealand Ministry of Business, Innovation, and Employment funding under High Value Manufacturing and Services (HVMS) Enabling Technologies investment contract C04X1205, Extrusion Plus.

\section{Availability of data and materials}

The datasets used and/or analyzed during the current study are available from the corresponding author on reasonable request.

\section{Declarations}

Competing interests

The authors declare that they have no competing interests.

Author details

${ }^{1}$ Scion, Rotorua, New Zealand. ${ }^{2}$ Polymer Standards Service, Mainz, Germany.

Received: 22 February 2021 Accepted: 15 July 2021

Published online: 26 July 2021

\section{References}

Andrianova AA, Yeudakimenka NA, Lilak SL, Kozliak El, Ugrinov A, Sibi MP, et al. Size exclusion chromatography of lignin: the mechanistic aspects and elimination of undesired secondary interactions. Journal of Chromatography A. 2018;1534:101-10. https://doi.org/10.1016/j.chroma.2017.12.051.

Baumberger S, Abaecherli A, Fasching M, Gellerstedt G, Gosselink R, Hortling B, et al. Molar mass determination of lignins by size-exclusion chromatography: towards standardisation of the method. Holzforschung. 2007;61(4):459-68. https://doi.org/10.1515/HF.2007.074. 
Cathala B, Saake B, Faix O, Monties B. Association behaviour of lignins and lignin model compounds studied by multidetector size-exclusion chromatography. J Chromatogr A. 2003;1020(2):229-39. https://doi.org/10.1016/j.chroma.2003. 08.046 .

Coffey A, Harmer R. Calibrating GPC columns - a guide to best practice. Agilent Technologies. 2015. https://www.agilent.com/cs/library/primers/public/ GPCstandardsPrimer_5991-2720EN.pdf. Accessed 01 Sep 2020.

Connors WJ, Sarkanen S, McCarthy JL. Gel chromatography and association complexes of lignin. Holzforschung. 1980;34(3):80-5. https://doi.org/10.1515/ hfsg.1980.34.3.80.

Constant S, Wienk HLJ, Frissen AE, Peinder PD, Boelens R, van Es DS, et al. New insights into the structure and composition of technical lignins: a comparative characterisation study. Green Chem. 2016;18(9):2651-65. https:// doi.org/10.1039/C5GC03043A

Contreras S, Gaspar AR, Guerra A, Lucia LA, Argyropoulos DS. Propensity of lignin to associate: light scattering photometry study with native lignins. Biomacromolecules. 2008;9(12):3362-9. https://doi.org/10.1021/bm800673a.

Dong D, Fricke AL. Intrinsic viscosity and the molecular weight of kraft lignin. Polym J. 1995;36(10):2075-8. https://doi.org/10.1016/0032-3861(95)91455-G.

Dubin PL, Koontz S, Wright KL III. Substrate-polymer interactions in liquid exclusion chromatography (GPC) in N,N-dimethylformamide. J Polym Sci Polym Chem Ed. 1977;15(9):2047-57. https://doi.org/10.1002/pol.1977.1701 50901.

Fredheim GE, Braaten SM, Christensen BE. Molecular weight determination of lignosulfonates by size-exclusion chromatography and multi-angle laser light scattering. J Chromatogr A. 2002;942(1-2):191-9. https://doi.org/10.1016/ s0021-9673(01)01377-2.

Froment P, Pla F. Lignin, properties and materials. Washington, DC: American Chemical Society; 1989.

Gellerstedt G. Gel permeation chromatography. In: Lin SY, Dence CW, editors. Methods in lignin chemistry. Berlin Heidelberg: Springer; 1992. p. 487-97. https://doi.org/10.1007/978-3-642-74065-7_34.

Gidh, AV, Decker SR, Vinzant TB, Himmel ME, Williford C. Determination of lignin by size exclusion chromatography using multi angle laser light scattering. J Chromatogr A. 2006;1114(1):102-10. https://doi.org/10.1016/j.chroma.2006.02. 044

Glasser WG, Dave V, Frazier CE. Molecular weight distribution of (semi-) commercial lignin derivatives. J Wood Chem Technol. 1993;13(4):545-59. https://doi.org/10.1080/02773819308020533.

Gosselink RJA, Abächerli A, Semke H, Malherbe R, Käuper P, Nadif A, et al. Analytical protocols for characterisation of sulphur-free lignin. Ind Crops Prod. 2004;19(3):271-81. https://doi.org/10.1016/j.indcrop.2003.10.008.

Grubisic Z, Rempp P, Benoit H. A universal calibration for gel permeation chromatography. J Polymer Sci B Polymer Lett. 1967;5(9):753-9. https://doi. org/10.1002/pol.1967.110050903.

Guerra A, Gaspar AR, Contreras S, Lucia LA, Crestini C, Argyropoulos DS. On the propensity of lignin to associate: a size exclusion chromatography study with lignin derivatives isolated from different plant species. Phytochemistry. 2007; 68(20):2570-83. https://doi.org/10.1016/j.phytochem.2007.05.026.

Hofe T. Tips \& Tricks GPC/SEC: how do I find the optimum sample concentration and injection volume for my GPC/SEC measurements?. 2007. https://www. pss-polymer.com/fileadmin/pdf/publication/TT_OptimumConcentration_E. pdf. Accessed 12 May 2021.

Huglin MB. Light scattering from polymer solutions. London, New York: Academic Press; 1972

Kuo C, Provder T, Koehler ME. GPC-viscometer study in THF, DMF and DMAC. In: Proceedings of International GPC Symposium; 1991. p. 47-159.

Lange H, Rulli F, Crestini C. Gel permeation chromatography in determining molecular weights of lignins: critical aspects revisited for improved utility in the development of novel materials. ACS Sustain Chem Eng. 2016;4(10): 5167-80. https://doi.org/10.1021/acssuschemeng.6b00929.

Liao JJ, Latif NAB, Trache D, Brosse N, Hussin MH. Current advancement on the isolation, characterization and application of lignin. International Journal of Biological Macromolecules. 2020;162:985-1024. https://doi.org/10.1016/j. ijbiomac.2020.06.168

Merkle G, Auerbach S, Burchard W, Lindner A, Wegener G. Light scattering of acetylated lignin. J Appl Polym Sci. 1992;45(3):407-15. https://doi.org/10.1 002/app.1992.070450305.

WinGPC Application Note. The WinGPC light scattering window (part 1) concentration method, PSS WinGPC Newsletter. 2010. https://www.psspolymer.com/infocenter/wingpc-newsletter. Accessed 13 May 2021.
Pellinen J, Salkinoja-Salonen M. High-performance size-exclusion chromatography of lignin and its derivatives. J Chromatogr A. 1985;328(Supplement C):299308. https://doi.org/10.1016/S0021-9673(01)87400-8.

Ringena O, Lebioda S, Lehnen R, Saake B. Size-exclusion chromatography of technical lignins in dimethyl sulfoxide/water and dimethylacetamide. J Chromatogr A. 2006;1102(1-2):154-63. https://doi.org/10.1016/j.chroma.2 005.10.037.

Siochi EJ, Ward TC, Haney MA, Mahn B. The absolute molecular weight distribution of hydroxypropylated lignins. Macromolecules. 1990;23(5):1420-9. https://doi.org/10.1021/ma00207a029.

Striegel AM, Yau WW, Kirkland JJ, Bly DD. Modern size-exclusion liquid chromatography. Practice of gel permeation and gel filtration chromatography, 2nd ed. Hoboken, New Jersey: Wiley; 2009.

Agilent Technologies (2017) A guide to multi-detector gel permeation chromatography. https://www.agilent.com/cs/library/primers/Public/5990-71 96EN.pdf. Accessed 12 May 2021

Tolbert A, Akinosho H, Khunsupat R, Naskar AK, Ragauskas AJ. Characterization and analysis of the molecular weight of lignin for biorefining studies. Biofuels, Bioproducts and Biorefining. 2014;8(6):836-56. https://doi.org/10.1 002/bbb.1500

Wang L, Shigetomi K, Koda K, Gele A, Uraki Y. A branched structure provides kraft lignins a denser morphology and a high molar mass for a given hydrodynamic radius. Holzforschung. 2020;74(6):551-8. https://doi.org/10.151 5/hf-2019-0292.

Wyatt PJ. Light scattering and the absolute characterization of macromolecules. Analytica Chimica Acta. 1993;272(1):1-40. https://doi.org/10.1016/0003-2 670(93)80373-S.

Zinovyev G, Sulaeva I, Podzimek S, Rossner D, Kilpelainen I, Sumerskii I, et al. Getting closer to absolute molar masses of technical lignins. ChemSusChem. 2018;11(18):3259-68. https://doi.org/10.1002/cssc.201801177.

\section{Publisher's Note}

Springer Nature remains neutral with regard to jurisdictional claims in published maps and institutional affiliations.

\section{Submit your manuscript to a SpringerOpen ${ }^{\circ}$ journal and benefit from:}

- Convenient online submission

- Rigorous peer review

- Open access: articles freely available online

- High visibility within the field

- Retaining the copyright to your article

Submit your next manuscript at $>$ springeropen.com 